\title{
Concepciones y prácticas de consumo y endeudamiento en adolescentes de la ciudad de Temuco, Chile ${ }^{1}$
}

\author{
Consumption and indebtedness practices of urban adolescents in schools from \\ Temuco
}

Solange Barros B., Valeria Cárdenas C., Marianela Denegri C. $^{2}$

Universidad de La Frontera, Chile

(ReCiBIDo 15/04/2015, AcEPTADo 20/06/2015)

\begin{abstract}
RESUMEN
La adolescencia constituye una etapa del desarrollo psicosocial que se ve influenciada por los contextos económico-político, social y cultural. Estos no solo inciden en la conformación de identidades sociales, sino también en la subjetividad y en las prácticas de consumo y endeudamiento de los[as] adolescentes. Aunque estos temas han sido de interés para la mercadotecnia, también constituyen un espacio de debate en los ámbitos de la psicología económica, social y del desarrollo. En este artículo se examinan las prácticas de consumo y endeudamiento de adolescentes escolarizados[as] urbanos[as] de la ciudad de Temuco en la Región de La Araucanía, Chile. Mediante un diseño de investigación cualitativa de tipo descriptivo, con diseño de casos múltiples, se realizaron grupos focales donde participaron 30 adolescentes, encontrándose diferencias en las prácticas de consumo y endeudamiento. Los resultados reportan la dimensión emocional implicada en casos de compras exitosas y frustradas, cuestionando a estudios anteriores que señalan déficits de previsión y ahorro de los[as] jóvenes.
\end{abstract}

Palabras clave: Adolescencia, consumo, endeudamiento, ahorro, emociones.

\begin{abstract}
The adolescence is a stage of psychosocial development that is influenced by economicpolitical, social and cultural contexts. They not only affect the formation of social identities, but also the subjectivity, consumption practices and indebtedness of adolescents. Although these issues have been of interest for marketing, they also hold a space for debate in the fields of economic, social and developmental psychology. This article describes the differences in consumption and indebtedness practices of urban adolescents in schools from Temuco, Region of La Araucania, Chile. Through a qualitative descriptive research design of multiple cases, focus groups were performed with 30 adolescents finding differences in consumption
\end{abstract}

1 Este trabajo fue financiado por el Proyecto Fondecyt № 1150665. Comisión Nacional de Investigación Científica y Tecnológica.

2 Docente del Departamento de Psicología, Universidad de La Frontera. E-mail: marianela.denegri@ufrontera.cl 
and borrowing practices. The results reported an emotional aspect involved in cases of successful and frustrated shopping, questioning previous studies that indicate deficits in the foresight and savings of these adolescents.

Keywords: Adolescence, consumption, indebtedness, savings, emotions.

En las últimas décadas, Chile se ha convertido en una referencia en cuanto a apertura a mercados extranjeros, experimentando importantes cambios a nivel de producción y tecnología, así como a nivel institucional y social, contribuyendo a generar un contexto sociopolítico y económico-cultural complejo y cambiante (Programa de las Naciones Unidas para el Desarrollo [PNUD], 1998). Uno de los cambios culturales relevantes se ha producido en los significados que la población atribuye al consumo y a sus prácticas, lo cual ha implicado una nueva configuración de los segmentos de consumidores y de sus necesidades y expectativas, impactando en la construcción de la identidad personal y social de los individuos (Denegri y Martínez, 2004).

En este contexto, no solo los adultos se involucran en las decisiones económicas de la familia, sino también niños y adolescentes, los cuales desde temprana edad comienzan a relacionarse con la realidad económica, a partir de experiencias cotidianas, como escuchar las frecuentes conversaciones que tienen sus padres y otros adultos sobre "lo económico" o ir al mercado (Amar, Llanos, Abello y Denegri, 2003). Por otra parte, las estrategias de mercadotecnia han ido cambiando de manera drástica, incorporando dentro de sus objetivos a nuevos segmentos que se caracterizan en muchos casos por no tener renta propia, pero sí un alto poder de influencia dentro de la familia, como es el caso de niños y jóvenes.

De esta manera, los jóvenes adolescentes son un segmento que resulta cada vez más de interés para el mercado, razón por la que muchas de las estrategias de mercadotecnia van orientadas a influir en sus patrones de consumo e impactar afectivamente en ellos. Este interés radica en sus particulares características como mercado de consumo, las cuales comparten con los niños, ya que de hecho aglutinan tres mercados distintos, uno presente porque comienzan a manejar dinero de forma autónoma a temprana edad, uno de influencia por su impacto en las decisiones de consumo de su familia, especialmente en productos tecnológicos y de ocio, y un mercado futuro en la medida que pueden ser fidelizados tempranamente (Denegri, Lara, Córdova y Del Valle, 2008). Este hecho es fundamental si se considera su etapa evolutiva de consolidación de la identidad donde se instalarán muchos de los patrones que les acompañarán en su vida adulta. Esta transcurre en un entorno donde el consumo se constituye en un articulador de los imaginarios sociales (Bauman, 2007), de tal forma que el acceso al consumo y a la posibilidad de compra y posesión de bienes deseados les permiten el acceso a símbolos y signos que favorecen la autorrealización y, además, favorecen la incorporación al grupo de pares (PNUD/INJUV, 2003). 
Por lo anterior, el proceso de construcción de identidad (personal, social y cultural) es un factor clave para comprender la aproximación al consumo en los adolescentes, dado que este se convierte en un vehículo de construcción de identidades ideales y en una forma de relación con otros que consideran "sus iguales" (Friedline, Elliot y Nam, 2012; Oyserman y Destin, 2010).

Según el Instituto Nacional de la Juventud, el 30\% de los jóvenes entre 15 y 29 años declara tener algún tipo de deuda (INJUV, 2012), mientras que del total de niños y adolescentes chilenos, un 3.8\% de entre 5 y 14 años y un $16.5 \%$ de los adolescentes de entre 15 y 17 realizan alguna actividad económica o tienen un trabajo (Encuesta de Actividades de Niños, Niñas y Adolescentes (EANNA), 2013). El mayor porcentaje de niños y adolescentes que trabaja o realiza alguna actividad económica se encuentra en el sur de Chile (10.1\%) (Ministerio de Desarrollo Social, 2013), aspecto importante si se considera que la región de la Araucanía, según la encuesta de caracterización socioeconómica [CASEN], (2011) es la región con el índice de pobreza más alto del país $(22.9 \%)$, con un $8.5 \%$ por sobre el promedio, lo cual se ve agravado si se considera que de la Región de la Araucanía, en Temuco, el $61 \%$ de la población señala estar endeudado. Lo anterior indica que existe un alto número de adolescentes que maneja dinero, por lo cual no son entes pasivos en la economía del país; influyen en el desarrollo de ella no solo a través de sus padres, sino también a través de su propia capacidad adquisitiva.

Respecto a las prácticas de consumo de los adolescentes, en Chile existen escasos estudios que permitan indagar en el perfil de los adolescentes como consumidores y en sus hábitos de uso del dinero. Uno de los escasos estudios previos es el de CNTV y McCann-Erikson (2005), que señala que independientemente del nivel socioeconómico, la mayoría de los adolescentes entre 13 y 17 años maneja dinero de forma regular (montos varían entre $\$ 5.000$ y $\$ 20.000$ ) y toman decisiones autónomas acerca de sus gastos, los cuales se orientan fundamentalmente a snacks, golosinas, música, videojuegos, ropa y accesorios. En este mismo estudio se observó que los adolescentes regularmente no destinan dinero al ahorro, e incluso el $55 \%$ declaró que le gustaría contar con más dinero para sus gastos.

Otra investigación acerca de la compresión del funcionamiento bancario, ahorro y endeudamiento en jóvenes de 14 a 18 años encontró que el conocimiento financiero de los adolescentes era muy rudimentario, observando diferencias por nivel socioeconómico, de tal forma que la comprensión de los adolescentes de los segmentos D y E era similar a la de niños menores de 10 años (Denegri, Martínez, Etchebarne, 2007).

Por su parte, en estudios que evalúan actitudes hacia el endeudamiento en la adolescencia, los jóvenes reportan una comprensión teórica sobre la importancia de la austeridad frente al consumo, como una protección ante el endeudamiento; no obstante, esto no se condice con sus prácticas de uso del dinero, puesto que estas aparecen poco sistematizadas, sin planificación y con escasa presencia de 
ahorro (Denegri, Cabezas, Páez, Sanhueza, Vargas, Zapata y Sepúlveda, 2010; Denegri, Lara, Córdova y Del Valle, 2008).

Por otra parte, un punto importante a tratar en los adolescentes es la emocionalidad asociada a la compra, ya que Bigné y Andreu (2004) afirman que los clientes con emociones positivas presentan mayor probabilidad de volver al centro comercial, que los clientes que no sienten esas emociones; además, estos autores plantean que cuando los consumidores sienten emociones positivas al comprar, muestran mayor satisfacción y lealtad hacia los entornos comerciales que frecuentan (Bigné y Andreu, 2004).

En síntesis, los antecedentes expuestos muestran que los adolescentes son un segmento que resulta cada vez más de interés para el mercado; y por las características de la etapa evolutiva de la adolescencia, en donde la construcción de identidad es una tarea prioritaria (Baumann, 2007), los coloca en una situación de riesgo futuro si no se considera su preparación para un manejo financiero racional que les permita mantener un equilibrio entre sus recursos y necesidades, ello porque las actitudes, hábitos y conductas económicas que se establecen en la adolescencia sustentarán gran parte del comportamiento económico adulto.

A partir de estas consideraciones, emerge la pregunta que orienta la presente investigación: ¿Cuáles son las concepciones y prácticas de consumo y endeudamiento en adolescentes escolarizados de la comuna de Temuco?

De acuerdo con esto, se propone como objetivo general: Caracterizar las prácticas de consumo y endeudamiento en adolescentes escolarizados de Temuco.

Como objetivos específicos se plantea:

- Describir las concepciones y prácticas de consumo en adolescentes escolarizados de Temuco.

- Describir las concepciones y prácticas de endeudamiento en los adolescentes participantes del estudio.

- Identificar las emociones asociadas a la compra en adolescentes escolarizados de Temuco.

\section{MÉTODO}

\section{Participantes}

Se utilizó un muestreo intencionado homogéneo. La muestra estuvo compuesta por 30 adolescentes, de ambos sexos, pertenecientes a establecimientos educacionales municipales y subvencionados de la ciudad de Temuco en el sur de Chile, distribuidos en 5 grupos focales de 6 adolescentes cada uno.

\section{Diseño}

Este estudio corresponde a una investigación cualitativa de tipo descriptivo, con diseño de casos múltiples. 


\section{Técnica}

La recolección de datos se realizó a través de grupos focales, para lo cual se utilizó un guion de entrevista semiestructurado que abordaba temas como el acceso al dinero, cantidades manejadas y quienes se las entregan, prácticas de consumo y de uso del dinero, endeudamiento temprano y emociones asociadas al acceso a compras deseadas.

\section{Procedimiento}

Los grupos focales se realizaron en los establecimientos a los que asistían los escolares, después de solicitar la autorización de la dirección de cada institución. Para mantener los resguardos éticos necesarios, se solicitó a los padres la firma de un consentimiento informado y a los estudiantes la firma de un asentimiento. Cada grupo contó con un facilitador entrenado para el diálogo con adolescentes, este hacía las preguntas base para estimular el diálogo y luego se estimulaba a todos los participantes a dar su opinión, cuidando que no se produjera una concentración excesiva en algunos de ellos. Los diálogos realizados en cada grupo fueron registrados electrónicamente y luego transcritos literalmente en un protocolo para su análisis.

Para el estudio de los datos se realizó un análisis cualitativo de contenido jerárquico ponderado, el cual tiene por finalidad la conceptualización del fenómeno en estudio a través de la caracterización diferencial de este, basándose en dimensiones de criterio usadas en la segmentación de la muestra.

De acuerdo con esto, inicialmente se realizó una codificación abierta de cada entrevista grupal a través del software para análisis de datos cualitativos Atlas ti, con el fin de representar con conceptos las ideas centrales, los cuales fueron comparados y revisados basados en la totalidad de los grupos focales. Posteriormente, se realizó una categorización y subcategorización de estos códigos y posteriormente fueron sistematizados en un dendograma que permite la organización visual de los resultados. Finalmente, se realizó un sumario porcentual de los códigos obtenidos, pues entrega el peso relativo de cada uno de estos.

\section{RESULTADOS}

Como se grafica en el dendograma de la figura 1, los resultados obtenidos se agruparon en tres núcleos centrales, el primero corresponde a las prácticas de consumo que presentan los adolescentes; el segundo se refiere a las prácticas de endeudamiento; y el tercero agrupa las emociones asociadas a la compra. 
CONSUMO Y ENDEUDAMIENTO EN ADOLESCENTES ESCOLARIZADOS DE TEMUC

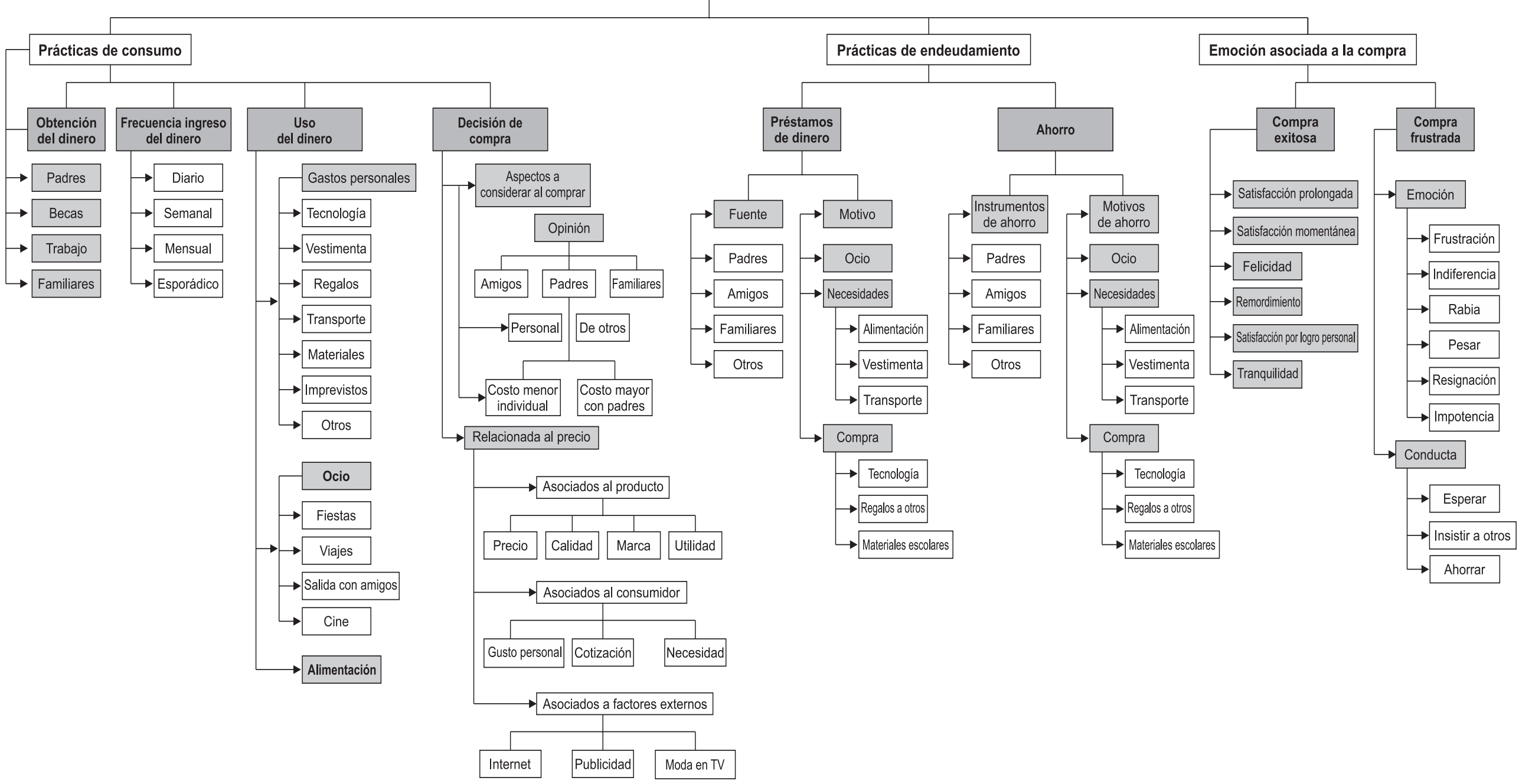

Figura 1. Dendograma. 


\section{Prácticas de consumo}

Los adolescentes participantes del estudio reportaron que las principales fuentes de obtención de dinero son los padres $(62.5 \%)$, becas $(16.7 \%)$, trabajo personal $(16.7 \%) \mathrm{y}$, en menor medida, los familiares $(4.2 \%)$. La frecuencia con que reciben estos ingresos es principalmente mensual $(42.9 \%)$, por medio de lo que los jóvenes denominan "mesada"; así también se observa que reciben dinero a diario (28.7\%), semanalmente (17.9\%) y de forma esporádica (17.9\%).

Respecto al uso del dinero, los adolescentes señalan invertir en gastos personales $(46.2 \%)$, ocio $(30.8 \%$ ) y alimentación $(23.1 \%)$; dentro de los primeros se encuentran gastos en transporte (47\%), vestimenta (13\%), imprevistos (13\%), materiales escolares $(10 \%)$, tecnología $(3 \%)$, regalos $(3 \%)$ y otros $(10 \%)$. Por su parte, en la categoría ocio se encuentran las salidas con amigos (70\%), viajes $(20 \%)$, cine $(20 \%)$ y, por último, fiestas $(5 \%)$.

En cuanto a la decisión de compra, los adolescentes refieren considerar la opinión de otros $(68 \%)$, opinión personal $(11 \%)$ y opinión relacionada al precio $(21 \%)$. En la primera categoría especifican que influye lo que opinen los padres (84\%), familiares (11\%) y amigos (5\%). En la segunda solo influye su opinión personal, y en la última categoría plantean que en la compra de productos de mayor costo influye la opinión de los padres $(50 \%)$, no así en la compra de artículos de menor costo, en donde la decisión es solo individual (50\%). Por otra parte, en la decisión de compra, los jóvenes también identifican que existen aspectos a considerar (68\%), los cuales están asociados al producto (47\%), asociados al consumidor $(30 \%)$ y asociados a factores externos $(23 \%)$.

Dentro de los asociados al producto se encuentra el precio $(50 \%)$, la calidad (32\%), la marca (14\%) y, en menor medida, la utilidad (4\%). En los elementos considerados asociados al consumidor, se destaca el gusto personal (44\%), la práctica de cotización (39\%) y la necesidad (17\%). Finalmente, los aspectos asociados a factores externos, y que influyen en la decisión de compra de los adolescentes, son la publicidad (57\%), la información de Internet (36\%) y la moda que muestran en televisión (7\%).

\section{Prácticas de endeudamiento}

Respecto a las prácticas de endeudamiento se encontró que los adolescentes solicitan préstamos de dinero (55\%), cuyas principales fuentes $(55.3 \%)$ son los padres $(43 \%)$, amigos $(33 \%)$, familiares $(24 \%)$ y otros $(5 \%)$ referidos a individuos cercanos al núcleo familiar. Los motivos (44.7\%) por los que solicitan los préstamos son para cubrir necesidades $(53 \%)$ de alimentación (56\%), transporte (33\%) y vestimenta (33\%); también utilizan estos recursos para comprar productos $(35 \%)$ como materiales escolares $(50 \%)$, tecnología (33\%) y regalos $(17 \%)$; y por último destinan el dinero solicitado como préstamo para solventar actividades de ocio $(12 \%)$. 
Como práctica antagónica al préstamo de dinero, los adolescentes reportaron llevar a cabo conductas de ahorro $(44.9 \%)$, dentro de ellas se encuentra que los principales instrumentos de ahorro (39\%), señalados por los participantes, son las tarjetas bancarias (50\%), alcancía (50\%), tenencia personal $(17 \%)$ y tenencia familiar (8\%). Por su parte, los motivos de ahorro (61.3\%) se relacionan con actividades de recreación (37\%), que se refieren a ocio $(57 \%)$, vacaciones $(29 \%)$ y salir con amigos (14\%). Otros motivos de ahorro son el cubrir gastos futuros $(32 \%)$ y realizar compras $(32 \%)$ en tecnología $(33 \%)$, regalos $(33 \%)$, calzado $(17 \%)$ y materiales escolares $(17 \%)$.

\section{Emoción asociada a la compra}

Dentro de los resultados, se evidenció que los adolescentes atribuyen emociones a la compra, diferenciando si esta fue exitosa (compraron lo que deseaban) o frustrada (no pudieron comprar el producto que deseaban).

De esta forma, en la compra exitosa (42\%) las principales emociones reportadas fueron la satisfacción prolongada (38\%), felicidad (19\%), satisfacción momentánea $(15 \%)$, satisfacción por logro personal (15\%) cuando el dinero es del joven, remordimiento por compras impulsivas $(8 \%)$ y tranquilidad $(4 \%)$.

Por otro lado, en las compras frustradas (58\%) se encontró una subdivisión que hace alusión a la emoción que les genera (72\%) y la conducta que llevan a cabo (27.8\%). Respecto a la emoción, se destaca la frustración (31\%), resignación $(31 \%)$, indiferencia $(12 \%)$, rabia $(12 \%)$, pesar $(8 \%)$ e impotencia $(8 \%)$. Por su parte, a nivel conductual se encuentra ahorrar para adquirir el producto $(60 \%)$, esperar ofertas $(30 \%) \mathrm{y}$, por último, insistir a otros para adquirir el producto $(10 \%)$.

\section{DISCUSIÓN}

Los resultados obtenidos muestran que el consumo y el endeudamiento están ampliamente generalizados entre los adolescentes.

Si bien la principal fuente de financiamiento son los padres, se observa que existen otras fuentes como el trabajo, las becas o el financiamiento de la familia extensa, lo cual les provee dinero de libre disposición, confirmando por lo tanto lo mencionado por Denegri, Lara, Córdova y Del Valle (2008), quienes afirman que los jóvenes comienzan a manejar dinero de forma autónoma a temprana edad e influyen ampliamente en las decisiones de consumo de su familia. Aunque este último aspecto no se indagó en las entrevistas realizadas, los participantes mencionan que en ocasiones critican las compras realizadas por sus familias: “...cuando mi mamá llega con algo y yo veo el precio... y costaba veinte mil pesos me enojo... le digo que lo devuelva porque a veces podemos encontrar la misma más barata”.

Mientras que respecto a la decisión de compra, los participantes afirman que la mayoría de las veces las compras las realizan en conjunto con sus padres, pero 
cada vez más se ve una tendencia a que compren y tomen decisiones por sí solos, lo cual se relaciona con la etapa evolutiva en la que se encuentran y concuerda con lo planteado por CNTV y McCann Erikson (2005) respecto a que los jóvenes tienden a tomar decisiones autónomas de sus gastos. Ahora bien, contrario a lo esperado, la influencia de los amigos no aparece como significativa en la presente investigación, lo cual no se condice con los antecedentes teóricos, en donde se señala que el consumo y la posibilidad de compra favorece la incorporación al grupo de pares, y por ende la opinión de estos constituiría un aspectos relevante a considerar al momento de consumir (PNUD/INJUV, 2003).

En relación con las prácticas de consumo, generalmente los adolescentes gastan su dinero en gastos personales como tecnología (principalmente celulares o computadores), vestimenta y regalos, así como en alimentación y ocio (salida con amigos y fiestas); CNTV y McCann Erikson (2005) se acercan en algunos aspectos en esta categoría al mencionar que los adolescentes entre 13 y 17 años manejan dinero regularmente y sus compras están orientadas fundamentalmente a snacks, golosinas, música, videojuegos, ropa y accesorios.

De igual modo, en este mismo aspecto se rescata una conducta de compra de los adolescentes, donde se destaca que en la compra de productos de un precio alto se realiza en conjunto con los padres, no así en productos que tienen un costo menor. Este aspecto es destacable a esta edad, ya que si bien son autónomos en algunas compras, aún queda un rezago de una conducta más infantil donde necesitan un asesoramiento de los adultos.

Por otro lado, se encontró que los adolescentes a la hora de comprar tienen en cuenta diversos aspectos, entre los cuales se hallan aquellos asociados al producto, como el precio, la calidad o la marca y aquellos asociados al consumidor, como la capacidad de cotización de los mismos y el gusto personal. Pero uno de los hallazgos más importantes, que confirma lo que investigaciones anteriores han propuesto, es la influencia que tiene el Internet o la publicidad en la compra. Estos resultados se asocian a lo que menciona Denegri, Lara, Córdova y Del Valle (2008), quienes afirman que los adolescentes son un segmento que resulta cada vez más de interés para el mercado, por lo que el marketing va orientado a influir en patrones de consumo impactando afectivamente en ellos.

Otro aspecto importante de mencionar es el alto endeudamiento presente en los adolescentes, pues un porcentaje significativo de los entrevistados habían pedido dinero prestado, en algunos casos sin devolución prevista, lo cual coincidió con lo reportado por el INJUV (2012), donde afirman que el 30\% de los jóvenes entre 15 y 29 años declara tener algún tipo de deuda, aunque se rescata que no reporta tener deudas con entidades bancarios o financieras, ya que las fuentes de endeudamiento son familiares o amigos.

Respecto a la capacidad de ahorro de los adolescentes, si bien no se indagó en si el ahorro es actual o pasado, la mayoría de los estudiantes afirma haber ahorrado, siendo sus formas de ahorro más comunes las tarjetas bancarias, las alcancías y la tenencia personal. Estos datos se diferencian de los encontrados en estudios an- 
teriores, donde se observó que los adolescentes regularmente no destinan dinero al ahorro e incluso el 55\% declara que le gustaría contar con más dinero para sus gastos (CNTV y McCann Erikson, 2005). De igual modo los adolescentes mostraron una adecuada comprensión de la importancia del ahorro, principalmente por la dificultad de sus padres para comprarles lo que desean.

Uno de los datos emergentes de este estudio y que pocas investigaciones anteriores han tratado se refiere a las emociones asociadas al consumo y que pueden influir directamente en las decisiones de compra. Entre las principales podemos encontrar la satisfacción y felicidad aunque algunos adolescentes afirman sentir remordimiento luego de comprar, principalmente por la falta de cotización, ya que creen que pudieron haber encontrado más barato el mismo producto. Las emociones influyen fuertemente en las decisiones de compra, pero no solo en aquello sino también en la fidelización de los clientes, ya que cuando los consumidores sienten emociones positivas muestran mayor satisfacción y lealtad hacia los entornos comerciales que frecuentan (Bigné y Andreu, 2004). Por lo cual es necesario poner atención en estos aspectos ya que se está creando un lazo comercial en una etapa evolutiva, en donde se está construyendo la identidad como tarea prioritaria (Bauman, 2007).

Por su parte, las emociones asociadas a cuando no pueden realizar las compras que desean se caracterizan principalmente por la frustración, la rabia y el pesar, de igual modo reconocen sentirse insatisfechos y con sentimientos de impotencia. En menor grado se puede encontrar la indiferencia y la aceptación a que la compra no se puede realizar. En cuanto a las conductas asociadas a una compra frustrada, son principalmente esperar a que el producto esté más barato o ahorrar, de lo cual se puede disgregar que el principal motivo por el que no se puede realizar una compra tiene que ver con motivos económicos.

Finalmente, dentro de los resultados, llama la atención que los adolescentes destinen dinero a imprevistos, e incluso reportan ahorrar para gastos futuros, lo cual evidencia que a pesar de lo expuesto en investigaciones anteriores, en donde se planteaba que las prácticas de consumo de los jóvenes tienden a ser poco sistematizadas, sin planificación y con escasa presencia de ahorro (Denegri; Cabezas, Páez, Sanhueza, Vargas, Zapata y Sepúlveda, 2010), los adolescentes en este estudio muestran conciencia de sus gastos e intentan llevar a cabo prácticas que les permitan suplir de forma adecuada necesidades futuras.

En conclusión, las prácticas de consumo de los adolescentes de Temuco se caracterizan por un financiamiento de los padres, mayoritariamente de manera mensual, donde el dinero se utiliza principalmente para el transporte diario y la salida con amigos. A la hora de comprar toman en cuenta en primer lugar la publicidad, posteriormente el precio y el gusto personal. Los adolescentes generalmente se endeudan con sus padres y amigos para alimentarse, comprar materiales escolares o pagar pasajes. En este estudio, se observaron prácticas de ahorro, las cuales se realizan en tarjetas bancarias o alcancías. Por su parte, la compra se caracteriza por una alta satisfacción por parte de los adolescentes, 
pudiendo ser esta prolongada o momentánea, mientras que cuando no pueden realizar la compra sienten frustración y resignación.

\section{CONCLUSIONES}

Si bien se encontraron prácticas similares a las observadas en estudios anteriores, es relevante destacar la presencia de ahorro y la visualización de gastos futuros, lo cual evidencia el inicio de un cambio de actitud frente al consumo y endeudamiento, debido posiblemente a la información a la que están expuestos los jóvenes y a la existencia de programas que buscan disminuir las conductas de riesgo respecto al endeudamiento.

La principal limitación de la presente investigación se relaciona con la falta de información respecto a variables sociodemográficas como el nivel socioeconómico y género de los participantes, ya que aquello habría permitido realizar análisis comparativos. De acuerdo con esto, se propone como futuras líneas de investigación realizar una comparación de las prácticas de consumo y endeudamiento según variables sociodemográficas como nivel socioeconómico y género, profundizar en el rol que juega el grupo de pares en las prácticas de consumo y endeudamiento de los adolescentes, y describir estas prácticas en población adolescente de sectores rurales.

\section{REFERENCIAS BIBLIOGRÁFICAS}

Amar, J., Llanos, M., Abello, R., y Denegri, M. (2003). Desarrollo del pensamiento económico en niños de la región caribe colombiana. Revista Latinoamericana de Psicología, 35, 1. 7-18. Recuperado de http://www. redalyc.org/pdf/805/80535101.pdf

Bauman, Z. (2007). Vida de consumo. Buenos Aires: Paidós.

Bigné, E. y Andreu, L. (2004). Emociones, satisfacción y lealtad del consumidor en entornos comerciales. Un análisis comparativo entre centro comercial y centro urbano. Departamento de Comercialización e Investigación de Mercados. Facultad de Economía, Universitat de València. Recuperado de http://www.mercasa.es/files/multimedios/1292349015 DYC_2004_76_77_87.pdf

CNTV y McCann, E. (2005). Informe 13.17 Adolescentes Chilenos. Recuperado desde http://www.cntv.cl/prontus_cntv/site/artic/20110415/asocfile/20110415102508/adolescentespresentaci_nfinal.pdf

Denegri, M., Cabezas, D., Páez, A., Sanhueza, O., Vargas, M., Zapata, L. y Sepúlveda, J. (2010). Actitudes hacia el endeudamiento en adolescentes de educación municipal y particular-subvencionada de la ciudad de Temuco. Revista de Educación y Humanidades, 1(2), 46-63. 
Denegri, M. (2004). Entrevista con Marianela Denegri. Endeudamiento y Consumo. Por Botero, M. Investigación en Psicología del consumidor (INPSICON). Recuperado de http://www.inpsicon.com/elconsumidor/archivos/entrevista_marianela_denegri.pdf

Denegri, M.; Lara, M.; Córdova; G. y Del Valle, C. (2008) Prácticas de ahorro $y$ uso del dinero en pre adolescentes (Tweens) chilenos. Universum, $\mathrm{N}^{\mathrm{o}}$ 23. Vol.1. 24 -39.

Denegri, M. y Martínez, G. (2004). ¿Ciudadanos o consumidores? Aportes constructivistas a la educación para el consumo. PAIDEIA. Revista de Educación, (37), 101-116.

Denegri, M., Martínez, G. y Etchebarne, S. (2007). La comprensión del funcionamiento bancario en adolescentes chilenos: un estudio de psicología económica. Interdisciplinaria, 2(24), 137-160.

Friedline, T., Elliott, W., \& Nam, I. (2012). Predicting savings and mental accounting among adolescents: The case of college. Children and Youth Services Review, 34(9), 1884-1895. doi:10.1016/j.childyouth.2012.05.018

Instituto Nacional de la Juventud (INJUV). (2012). Encuesta nacional de juventud 2012. Recuperado de http://www.injuv.gob.cl/portal/wp-content/ uploads/2013/08/SÉPTIMA-ENCUESTA-NACIONAL-JUVENTUD-2. pdf

Ministerio de Desarrollo Social (2012). CASEN 2011, Encuesta de caracterización socioeconómica nacional. Recuperado de http://observatorio. ministeriodesarrollosocial.gob.cl/layout/doc/casen/publicaciones/2011/ CASEN_2011.pd

Ministerio de Desarrollo Social. (2013). Principales resultados: Encuesta de actividades de niños, niñas y adolescentes (EANNA) 2012. Recuperado de http://observatorio.ministeriodesarrollosocial.gob.cl/layout/doc/eanna/ presentacion_EANNA_28junio_final.pdf

PNUD/INJUV. (2003). Transformaciones Culturales e Identidad Juvenil en Chile. Santiago: PNUD/INJUV.

Programa de las Naciones Unidas para el Desarrollo (PNUD). (1998). Informe de desarrollo humano. Recuperado de http://hdr.undp.org/sites/default/ files/hdr_1998_es_completo_nostats.pdf

Programa de las Naciones Unidas para el Desarrollo (PNUD) (2012). Desarrollo humano en Chile. Bienestar subjetivo: el desafio de repensar el desarrollo. Recuperado de http://www.desarrollohumano.cl 